\title{
Mehr Schlaganfälle bei hoher Feinstaubbelastung
}

\section{Luftverschmutzung belastet nicht nur die Atemwege. Ein Anstieg der Feinstaubbelastung in der Luft führte in einer amerikanischen Studie bereits nach wenigen Stunden zu einem erhöhten Schlaganfallrisiko.}

- In die retrospektive Beobachtungsstudie wurden 1705 Patienten aufgenommen, die zwischen 1999 und 2008 wegen eines akuten ischämischen Schlaganfalls in einem Krankenhaus in Boston stationär behandelt wurden. Während des gleichen Zeitraums wurde die Konzentration von Feinstaub $<2,5 \mu \mathrm{m}$ (PM 2,5) und anderen Substanzen in der Umgebungsluft an der nahegelegenen Messstation der Harvard University kontinuierlich gemessen. Alle Patienten lebten im Umkreis von 40 $\mathrm{km}$ um diese Messstation.

Es zeigte sich, dass das Schlaganfallrisiko während einer 24-Stunden-Periode mit moderater Feinstaubkonzentration $\left(15-40 \mu \mathrm{g} / \mathrm{m}^{3}\right)$ um $34 \%$ erhöht war im Vergleich zu 24-Stunden-Perioden mit geringer Feinstaubkonzentration $(\leq 15 \mu \mathrm{g} /$ $\mathrm{m}^{3}$ ). Über den gesamten Bereich der gemessenen Feinstaubkonzentrationen hinweg fand sich ein Anstieg des Schlaganfallrisikos um $11 \%$ pro Zunahme der Konzentration um $6,4 \mu \mathrm{g} / \mathrm{m}^{3}$.

Ähnliche Zusammenhänge mit dem Schlaganfallrisiko fanden sich auch für höhere Konzentrationen von Ruß und Stickstoffdioxid, nicht aber für andere Luftschadstoffe.

- Wellenius GA et al.

Ambient air pollution and the risk of acute ischemic stroke. Arch. Intern. Med. 172 (2012) 229-234

\section{Kommentar}

Es ist mittlerweile gut belegt, dass eine erhöhte Feinstaubbelastung der Umgebungsluft zahlreiche Gesundheitsschäden auslöst und neben Atemwegserkrankungen auch das Risiko für Herzinfarkt, arterielle Verschlusskrankheit, Mortalität und Krankenhausbehandlung erhöht. Die vorliegende Studie belegt, dass bei höheren Feinstaubbelastungen auch das Risiko eines Schlaganfalls ansteigt. Ursächlich können dabei inflammatorische, hämostatische, endotheliale und vegetative Prozesse eine Rolle spielen.

Die Tatsache, dass neben der Feinstaubkonzentration auch erhöhte Konzentrationen von Ruß und Stickstoffdioxid das Schlaganfallrisiko erhöhten, deutet nach Einschätzung der Autoren vor allem auf die Bedeutung des Straßenverkehrs als Hauptquelle der Schädigung hin. Auch wenn die individuelle Risikoerhöhung für den Einzelnen dabei gering sein mag, ergibt sich aufgrund der sehr weiten Verbreitung erhöhter Feinstaubkonzentrationen und anderer Luftbelastungen durch Straßenverkehr auf Bevölkerungsebene ein hohes populationsattributables Risiko.

\section{Achtung:}

\section{Hier muss der Dummy durch eine Anzeige ersetzt werden !!}

